FRIDAY, 16 JUNE 2017

Innovative insights into mechanism of SLE, Sjögren's and APS

\section{OP0299 SERUM AND GLOMERULAR EXPRESSION OF IL32 IN LUPUS NEPHRITIS}

S. Truglia ${ }^{1}$, C. Alessandri ${ }^{1}$, F. Ciccia ${ }^{2}$, A. Rizzo ${ }^{2}$, T. Colasanti $^{3}$, F. Miranda ${ }^{3}$ F.R. Spinelli ${ }^{3}$, F. Ceccarelli ${ }^{3}$, G. Triolo ${ }^{2}$, A. Capozzi ${ }^{4}$, M. Sorice ${ }^{4}$, G. Valesini ${ }^{3}$, F. Conti ${ }^{3} .{ }^{1}$ Medicina Interna e Specilità Mediche, Reumatologia, Sapienza Università di Roma, Roma: ${ }^{2}$ Dipartimento Biomedico di Medicina Interna e Specialistica, Sezione di Reumatologia, Università di Palermo, Palermo ${ }^{3}$ Dipartimento di Medicina Interna e Specialità Mediche, Reumatologia; ${ }^{4}$ Dipartimento di Medicina Sperimentale, Sapienza Università di Roma, Roma, Italy

Background: Lupus nephritis (LN) is one of the most severe features of systemic lupus erythematosus (SLE). Several cytokines and chemokines are secreted locally in case of glomerular inflammation. Interleukin 32 (IL32) is a newly described cytokine that exhibits several properties typical of proinflammatory cytokines. Ex vivo and in vitro studies supported the role of Toll like receptors (TLRs) in LN pathogenesis and recent investigations demonstrated that Poly I:C, a ligand for (TLR) 3, strongly induced IL32 production from several cell populations. Objectives: To investigate serum and urinary levels of IL32 in a cohort of LN patients compared to SLE patients without renal involvement and healthy controls (HC). In addition, we investigated kidney expression of IL32 and in vitro ability of LN patients' serum IgG to stimulate IL32 production by TLR3 activation in human embryonic kidney 293 cells line stably transfected with a TLR3 plasmid (Hek293/T3).

Methods: Serum and urinary IL32 concentrations were measured using ELISA; a polyclonal rabbit anti-human IL32 was used to evaluate the expression of IL32 in renal biopsies. To assess the production of IL32 induced by patients' IgG and the transduction pathway of TLR3 we performed Western Blot analysis for IL32, TBK1 and NFkB.

Results: We recruited $60 \mathrm{LN}$ patients, 50 SLE patients without renal involvement and $30 \mathrm{HC} ; 40 \mathrm{LN}$ patients had an active disease (a-LN) and the remaining 20 were in remission ( $r$-LN). IL32 serum levels were significantly higher in patients with r-LN (median 1368, IQR 3910) and HC (median 721, IQR 2271) compared to SLE patients without renal involvement (median 203, IQR $662.8 \mathrm{pg} / \mathrm{ml})(\mathrm{p}=0.03$ and $p=0.018$, respectively). There were no significant differences in urinary IL32 levels among LN patients, SLE patients without renal involvement and HC. In LN patients a direct association between IL32 serum levels and disease duration $(p=0.02 ; r 0.2978)$ was observed. Immuno-histochemical analysis performed on renal biopsies of 20 a-LN and $8 \mathrm{HC}$ showed that IL32 was strongly expressed in renal samples of LN patients, especially in patients with class IV, compared to controls. Western Blot analysis showed that antibodies isolated from LN patients induced in vitro production of IL32 in Hek293/T3 cells as well as the phosphorylation of NFkB and TBK1.

Conclusions: The results of the present study show increased IL32 serum levels in $r$-LN patients as well as increased expression of such cytokine in renal tissues of a-LN patients. IL32 renal expression and its production by Hek293/T3 cells after patients' IgG stimulation, probably mediated by TLR3, may suggest the production of IL32 directly at renal level in course of LN.

Disclosure of Interest: None declared

DOI: 10.1136/annrheumdis-2017-eular.5277

\section{OP0300 ENRICHMENT OF T FOLLICULAR-HELPER CELLS (TFH) AND EXCLUSION OF T FOLLICULAR-REGULATORY CELLS (TFR) FROM ECTOPIC GERMINAL CENTERS IN SALIVARY GLANDS OF SJOGREN'S SYNDROME PATIENTS}

E. Pontarini ${ }^{1}$, W. Murray-Brown ${ }^{1}$, C. Croia ${ }^{1}$, E. Astorri ${ }^{1}$, D. Lucchesi ${ }^{1}$ N. Lepse ${ }^{1}$, N. Sutcliffe ${ }^{2}$, A. Tappuni ${ }^{3}$, C. Pitzalis ${ }^{1}$, M. Bombardieri ${ }^{1} .{ }^{1}$ Centre for Experimental Medicine \& Rheumatology, William Harvey Research Institute; ${ }^{2}$ Barts Health; ${ }^{3}$ Institute of Dentistry, Queen Mary University of London, London, United Kingdom

Background: $B / T$ cell aggregates in the salivary glands (SG) of Sjögren's syndrome (SS) can give rise to ectopic lymphoid structures (ELS) forming ectopic germinal centers (GCs), which has been linked to the development of MALT lymphoma (MALT-L). T follicular-helper cells (Tfh) and T follicular-regulatory cells (Tfr) are specialized CD4+ T-cells that positively and negatively regulate, respectively, the magnitude of the GCs response and the onset of autoimmunity. Objectives: To characterize the infiltration of Tfh and Tfr in the SG infiltrates of patients with SS in the context of the presence/absence of ectopic GCs and in subjects with MALT-L.

Methods: SG biopsies with matching histology and RNA from 37 SS and 38 nonspecific chronic sialadenitis (NSCS) patients were stratified as ELS-/ELS+ based on $\mathrm{CD} 3 / \mathrm{CD} 20 / \mathrm{CD} 21 / \mathrm{CD} 138$ immunostaining (IHC). Histological samples and mRNA from 12 parotid MALT-L were also studied. Gene expression was measured by Taqman rt-PCR. Multicolor immunofluorescence/confocal microscopy for CD3, CD4, CD45RO, ICOS, PD1, BCL6 and FoxP3 was used to identify Tfh and Tfr.
Results: Tfh $\left(\mathrm{CD}^{+} \mathrm{CD}^{2} 5 \mathrm{RO}^{+} \mathrm{PD} 1^{+} \mathrm{ICOS}^{+} \mathrm{FoxP}^{-}\right)$cells and $\mathrm{Tfr}$ $\left(\mathrm{CD} 4^{+} \mathrm{CD} 45 \mathrm{RO}^{+} \mathrm{PD}^{+}{ }^{+} \mathrm{ICOS}^{+} \mathrm{FoxP}^{+}\right)$cells were strongly enriched in ELS+ vs ELS- SS samples. The Tfh:Tfr ratio in ELS+ SG was approximately 2:1. Interestingly, while in tonsils Tfr were routinely detected within GCs, in ELS+ SG Tfr were predominantly excluded from the B cell follicles and accumulated in the $T$ cell rich areas at the periphery of the lymphoid aggregates. Conversely, Tfh densely infiltrated the B cell rich areas and, within ectopic GCs, acquired BCL6. Furthermore, Thh infiltration closely correlated with SG IL-21 mRNA expression, which in turn was strongly correlated with CD3, CD20 and CD138 IHC scores and with CXCL13, LTb, BAFF, AID and Pax5 gene expression. Finally, MALT-L samples displayed 10 -fold higher IL-21 mRNA and twice as much PD $1^{+} I C O S^{+} B C L 6^{+}$ Tfh-cells/field compared to ELS+ SS samples.

Conclusions: Within the SG of SS patients Tfh cells closely segregate with lesional IL-21 expression, localize within ELS and are strongly enriched during MALT-L development. Conversely, although Tfr cells are also recruited to ELS+ SG in SS patients, we consistently demonstrated follicular exclusion of this subset from ectopic GCs. This suggests that Tfr in SS SG fail to exert their physiological immunoregulatory properties in controlling the magnitude of the GCs response and $B$ cell autoreactivity, as observed in tonsils.

Acknowledgements: This work was supported by project grants from the Medical Research Council (MR/N003063/1 to MB) and Arthritis Research UK (grant 20089 to MB).

Disclosure of Interest: E. Pontarini: None declared, W. Murray-Brown: None declared, C. Croia: None declared, E. Astorri: None declared, D. Lucchesi: None declared, N. Lepse: None declared, N. Sutcliffe: None declared, A. Tappuni: None declared, C. Pitzalis: None declared, M. Bombardieri Consultant for: Amgen/Medimmune, GSK and UCB

DOI: 10.1136/annrheumdis-2017-eular.6121

\section{OP0301 TYPE I IFN GENE SIGNATURE TEST-HIGH AND -LOW PATIENTS WITH MODERATE TO SEVERE SLE DISEASE ACTIVITY HAVE DISTINCT GENE EXPRESSION SIGNATURES OF IMMUNOLOGIC PATHWAYS AND CELL TYPES}

H. Liu ${ }^{1}$, B. Higgs ${ }^{1}$, W. Rees ${ }^{1}$, C. Morehouse ${ }^{1}$, K. Streicher ${ }^{1}$, P. Brohawn ${ }^{2}$, G. Illei ${ }^{1}$, K. Ranade ${ }^{1} .{ }^{1}$ Medlmmune LLC; ${ }^{2}$ Medlmmune, Gaithersburg, United States

Background: Type I interferon (IFN) has been implicated in systemic lupus erythematosus (SLE) pathogenesis, and the majority of patients with SLE have elevated expression of type I IFN-inducible genes in their blood. Anifrolumab, a fully human, $\lg \mathrm{G}_{1} \kappa$ monoclonal antibody against the type I IFN receptor, is in Phase III development for the treatment of moderate to severe SLE (NCT02446912 and NCT02446899)

Objectives: We sought to understand other molecular pathways (either dependent on or independent of type I IFN signaling), to elucidate heterogeneous mechanisms in SLE, and to identify patient subsets for personalized disease management.

Methods: Baseline blood samples from adult patients with moderate to severe SLE from two Phase llb clinical studies (NCT01438489, N=265; NCT01283139, $\mathrm{N}=416$ ) were profiled with whole genome array analyses. Type I IFN gene signature (IFNGS) test status was determined by a central laboratory utilizing an analytically validated four gene (IFI27, IFI44, IFI44L, RSAD2) quantitative polymerase chain reaction-based test from patients' whole blood. A predetermined, delta Ct-based cut-off point, in the trough of the bimodal distribution, was utilized to segregate type I IFNGS test-high from -low patients at baseline. Blood from healthy controls was stimulated ex vivo with IFN- $\beta$, IFN- $\gamma$, IFN- $\lambda$, IFN- $\omega$, or a pool of all IFN- $\alpha$ subtypes, with or without blocking antibodies for each IFN type, to develop IFN-type-specific signatures. Cell type- and cytokine pathway-specific gene signatures derived from the literature were also evaluated with the Phase Ilb sample data. A Fisher's exact test was used for enrichment calculations (signatures cut at median), and comparisons were adjusted for multiplicity through false discovery rate.

Results: A total of $79 \%$ of SLE patients in the combined study population had a type I IFNGS test-high status. From the type I IFNGS test-high patients, 29/95 signatures evaluated had significant enrichment, including those for $B$ cells $(q=1.17 \mathrm{E}-17$, odds ratio $[\mathrm{OR}]=6.4)$, plasma cells $(\mathrm{q}=6.96 \mathrm{E}-11, \mathrm{OR}=3.9)$, and $\mathrm{CD} 40 \mathrm{~L}$ signaling $(\mathrm{q}=1.07 \mathrm{E}-08, \mathrm{OR}=3.3$ ), relative to type I IFNGS test-low patients. In contrast, type I IFNGS test-low patients had enrichment for eosinophils $(\mathrm{q}=5.4 \mathrm{E}-6, \mathrm{OR}=0.39)$ and type II IFN (IFN- $\gamma)$ specifically inducible gene signatures $(\mathrm{q}=4.6 \mathrm{E}-3, \mathrm{OR}=0.47)$. These findings were significant for the combined study population, as well as for the NCT01438489 study population, and were either significant or trending for the NCT01283139 population $(q<0.05)$.

Conclusions: SLE patients who are type I IFNGS test-high had elevated concentrations of $B$ cells, plasma cells, and other inflammatory cytokine pathways. Type I IFNGS test-low patients, by contrast, were enriched for eosinophil and type II IFN pathways. These observations provide new insights into the molecular heterogeneity underlying SLE and suggest new therapeutic approaches, particularly for type I IFNGS test-low patients.

Acknowledgements: Funded by Medlmmune. Medical writing support was provided by R. Plant, QXV Comms, an Ashfield business, UK.

Disclosure of Interest: H. Liu Employee of: Medlmmune LLC, B. Higgs Shareholder of: AstraZeneca, Employee of: Medlmmune LLC, W. Rees Employee of: Medlmmune LLC, C. Morehouse Shareholder of: AstraZeneca, Employee of: Medlmmune LLC, K. Streicher Employee of: Medlmmune LLC, P. Brohawn 